\title{
Reliability analysis based on gradient and heuristic optimization techniques of composite laminates using element-free Galerkin method
}

\author{
J.E. Rojas ${ }^{1,2, a}$, A. El Hami and D.A. Rade ${ }^{2}$ \\ 1 Institut National des Sciences Appliquées de Rouen, Laboratoire de Mécanique de Rouen, BP 08, Avenue de l’Université, \\ 76801 Saint-Etienne du Rouvray, Cedex, France \\ 2 Federal University of Uberlândia, School of Mechanical Engineering, 2121, Av. Joao Naves de Avila, Campus Santa Mônica, \\ CEP 38400-902 CP 593, Uberlândia, Brazil
}

Received 27 December 2007, accepted 25 March 2008

\begin{abstract}
This work presents a reliability methodology that couples gradient and heuristic based reliability methods with element-free Galerkin method applied in composite laminates structures. First and second order reliability methods are the gradient based reliability methods. The heuristic based reliability method suggested by authors uses natured-inspired optimization method: genetic algorithms. Numerical examples on linear elasticity that involve random properties of laminated composite plates are presented in order to demonstrate the efficiency, capacity and robustness of the proposed methodology. The results show that the predicted reliability levels are accurate in comparison with similar approach that uses finite element analysis to evaluate implicit limit state functions.
\end{abstract}

Key words: Composite laminates; element-free Galerkin method; first order and second order reliability methods; heuristic based reliability method.

\section{Introduction}

Classical mesh-based methods, such as finite elements, depend clearly on their dependence on a mesh in the simulations of mechanical problems. In this sense, meshless methods have been proposed to eliminate the mesh dependent structure, of the classical mesh-based methods, by constructing the approximation entirely in terms of point, without element connectivity. From principles of ninety years, the meshless methods have experiment a fort computational development in solid mechanics. However, most of meshless methods have been focused on deterministic analysis [1]. In consequence, the research in meshless methods considering probabilistic analysis had not received much attention. The developed meshless method provides a rich, relatively unexplored area for research in computational structural reliability analysis.

Among meshless methods, element-free Galerkin (EFG) method is particularly appealing due to their simplicity and a formulation that corresponds to the wellestablished finite element method (FEM) [2]. The EFG method uses moving least squares approximation to construct shape functions based on a set of nodes scattered in the problem domain. The essential boundary conditions are imposed using their weak forms with Lagrange multipliers.

\footnotetext{
${ }^{a}$ Corresponding author: jhojan_enrique@yahoo.es
}

In this work the EFG method is used in structural reliability analysis for simulate laminated composite plates on linear elasticity. The reliability procedure proposed in this work, it is based on gradient and heuristic based reliability methods for estimate the reliability index. First and second order reliability methods (FORM and SORM, respectively) are the gradient based reliability methods which uses Newton-Raphson (N-R) procedure. The heuristic based reliability method (HBRM) suggested in [3] uses natured-inspired optimization methods: genetic algorithms (GA) [4,5], particle swarm optimization (PSO) $[6,7]$ and ant colony optimization (ACO) $[8,9]$. In this work, GA it is used in numerical applications. When compared with FORM and SORM methods, HBRM it is different because not requires the initial guess and the computation of gradients of the limit state function because is based on multidirectional search.

The obtained results are compared with FEM solutions in order to evaluate the efficiency, capacity and robustness of the proposed methodology.

\section{Stress-strain relations for plane stress in an orthotropic material}

Consider the composite laminated plate shown in Figure 1 using a Cartesian coordinate system. The fibre orientation of a layer in indicated by $\theta$ as shown in Figure 1. 


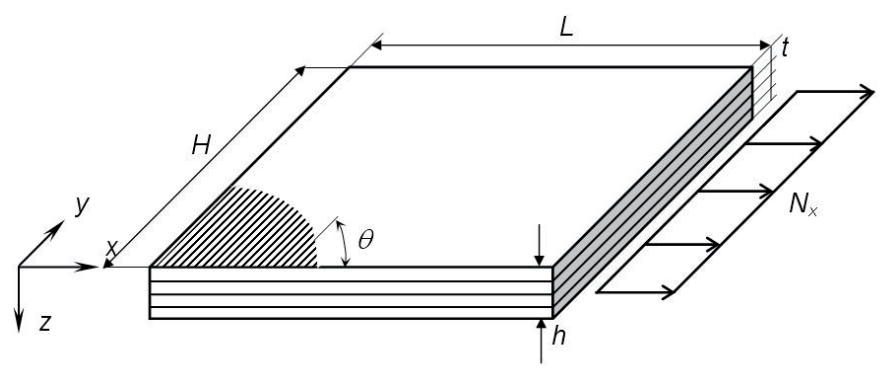

Fig. 1. Laminated composite plate and its fibre orientation.

The generalized Hooke's law for $k$ layer relating stresses to strains can be expressed as follows [10]:

$$
\{\sigma\}_{k}=[\bar{Q}]_{k}\{\varepsilon\}_{k},
$$

where $\{\sigma\}$ is the vector of stress components, $[\bar{Q}]$ is the stiffness matrix, and $\{\varepsilon\}$ is the strain components.

From classical lamination theory, based on Kirchhoff's assumptions, the kinematics of laminated composites are given by:

$$
\begin{aligned}
&\left\{\begin{array}{c}
\varepsilon_{x} \\
\varepsilon_{y} \\
\gamma_{x y}
\end{array}\right\}=\left\{\begin{array}{c}
\varepsilon_{x}^{0} \\
\varepsilon_{y}^{0} \\
\gamma_{x y}^{0}
\end{array}\right\}+z\left\{\begin{array}{c}
k_{x} \\
k_{y} \\
2 k_{x y}
\end{array}\right\}= \\
&\left\{\begin{array}{c}
\frac{\partial u_{0}}{\partial x} \\
\frac{\partial v_{0}}{\partial y} \\
\frac{\partial u_{0}}{\partial y}+\frac{\partial v_{0}}{\partial x}
\end{array}\right\}+z\left\{\begin{array}{c}
\frac{\partial^{2} w_{0}}{\partial x^{2}} \\
\frac{\partial^{2} w_{0}}{\partial y^{2}} \\
2 \frac{\partial^{2} w_{0}}{\partial x \partial y}
\end{array}\right\}
\end{aligned}
$$

where $u_{0}, v_{0}$ and $w_{0}$ are the displacements of the middle plane in the $x, y$ and $z$ directions, respectively. are denoted by $u, v, w$, respectively; $k_{x y}$ is the total skew curvature.

The constitutive equations for $k$ layer can be written as follows [10]:

$$
\left\{\begin{array}{l}
\sigma_{x} \\
\sigma_{y} \\
\tau_{x y}
\end{array}\right\}_{k}=[T]_{k}^{-1}[Q]_{k}[T]_{k}^{T}=\left[\begin{array}{lll}
\bar{Q}_{11} & \bar{Q}_{12} & \bar{Q}_{16} \\
\bar{Q}_{12} & \bar{Q}_{22} & \bar{Q}_{26} \\
\bar{Q}_{16} & \bar{Q}_{26} & \bar{Q}_{66}
\end{array}\right]_{k}\left\{\begin{array}{l}
\varepsilon_{x} \\
\varepsilon_{y} \\
\gamma_{x y}
\end{array}\right\}_{k},
$$

where:

$$
\begin{aligned}
\bar{Q}_{11}= & Q_{11} \cos ^{4} \theta+2\left(Q_{12}+2 Q_{66}\right) \sin ^{2} \theta \cos ^{2} \theta+Q_{22} \sin ^{4} \theta \\
\bar{Q}_{12}= & \left(Q_{11}+Q_{22}-4 Q_{66}\right) \sin ^{2} \theta \cos ^{2} \theta \\
& +Q_{12}\left(\sin ^{4} \theta+\cos ^{4} \theta\right) \\
\bar{Q}_{22}= & Q_{11} \sin ^{4} \theta+2\left(Q_{12}+2 Q_{66}\right) \\
& \times \sin ^{2} \theta \cos ^{2} \theta+Q_{22} \cos ^{4} \theta \\
\bar{Q}_{16}= & \left(Q_{11}-Q_{22}-2 Q_{66}\right) \sin \theta \cos ^{3} \theta \\
& +\left(Q_{12}-Q_{22}+2 Q_{66}\right) \sin ^{3} \theta \cos \theta \\
\bar{Q}_{26}= & \left(Q_{11}-Q_{22}-2 Q_{66}\right) \sin ^{3} \theta \cos \theta \\
& +\left(Q_{12}-Q_{22}+2 Q_{66}\right) \sin \theta \cos 3 \\
\bar{Q}_{66}= & \left(Q_{11}+Q_{22}-2 Q_{12}-2 Q_{66}\right) \sin ^{2} \theta \cos ^{2} \theta \\
& +Q_{66}\left(\sin ^{4} \theta+\cos ^{4} \theta\right) .
\end{aligned}
$$

In the last equations: $\nu_{12} E_{2}=\nu_{21} E_{1}, Q_{66}=G_{12}$, $Q_{11}=E_{1} /\left(1-\nu_{12} \nu_{21}\right), Q_{22}=E_{2} /\left(1-\nu_{12} \nu_{21}\right)$ and $Q_{12}=\nu_{12} E_{2} /\left(1-\nu_{12} \nu_{21}\right)=\nu_{21} E_{1} /\left(1-\nu_{12} \nu_{21}\right)$ where $G_{12}$ is the shear modulus, $E_{1}$ and $E_{2}$ are Young's modulus parallel and perpendicular to the fibres while $\nu_{12}$ and $\nu_{21}$ are the corresponding Poisson's ratios.

The coordinate transformation matrix is [11]:

$$
[T]=\left[\begin{array}{ccc}
\cos ^{2} \theta & \sin ^{2} \theta & 2 \sin \theta \cos \theta \\
\sin ^{2} \theta & \cos ^{2} \theta & -2 \sin \theta \cos \theta \\
-\sin \theta \cos \theta & -\sin \theta \cos \theta & \cos ^{2} \theta-\sin ^{2} \theta
\end{array}\right] .
$$

The relations between the resultants (forces $N$ and moments $M$ ) and the strains (strains $\varepsilon$ and curvatures $\mathbf{k}$ ) are given by:

$$
\begin{aligned}
& \left\{\begin{array}{l}
N_{x} \\
N_{y} \\
N_{x y}
\end{array}\right\}=\left[\begin{array}{lll}
A_{11} & A_{12} & A_{16} \\
A_{12} & A_{22} & A_{26} \\
A_{16} & A_{26} & A_{66}
\end{array}\right]\left\{\begin{array}{c}
\varepsilon_{x}^{0} \\
\varepsilon_{y}^{0} \\
\gamma_{x y}^{0}
\end{array}\right\} \\
& +\left[\begin{array}{lll}
B_{11} & B_{12} & B_{16} \\
B_{12} & B_{22} & B_{26} \\
B_{16} & B_{26} & B_{66}
\end{array}\right]\left\{\begin{array}{l}
k_{x} \\
k_{y} \\
2 k_{x y}
\end{array}\right\}, \\
& \left\{\begin{array}{l}
M_{x} \\
M_{y} \\
M_{x y}
\end{array}\right\}=\left[\begin{array}{lll}
B_{11} & B_{12} & B_{16} \\
B_{12} & B_{22} & B_{26} \\
B_{16} & B_{26} & B_{66}
\end{array}\right]\left\{\begin{array}{l}
\varepsilon_{x}^{0} \\
\varepsilon_{y}^{0} \\
\gamma_{x y}^{0}
\end{array}\right\} \\
& +\left[\begin{array}{lll}
D_{11} & D_{12} & D_{16} \\
D_{12} & D_{22} & D_{26} \\
D_{16} & D_{26} & D_{66}
\end{array}\right]\left\{\begin{array}{l}
k_{x} \\
k_{y} \\
k_{x y}
\end{array}\right\} .
\end{aligned}
$$

Combine the above equations:

$$
\left\{\begin{array}{l}
\mathbf{N} \\
\mathbf{M}
\end{array}\right\}=\left[\begin{array}{ll}
\mathbf{A} & \mathbf{B} \\
\mathbf{B} & \mathbf{D}
\end{array}\right]\left\{\begin{array}{l}
\varepsilon \\
\mathbf{k}
\end{array}\right\}
$$

where $\mathbf{A}$ is called the extensional stiffness matrix, $\mathbf{B}$ is called the coupling stiffness matrix and $\mathbf{D}$ is called the bending stiffness matrix of the laminate. The components of these three matrices are defined as follows:

$$
\begin{aligned}
& A_{i j}=\sum_{k=1}^{n}\left(\bar{Q}_{i j}\right)_{k}\left(z_{k}-z_{k-1}\right) \\
& B_{i j}=\frac{1}{2} \sum_{k=1}^{n}\left(\bar{Q}_{i j}\right)_{k}\left(z_{k}^{2}-z_{k-1}^{2}\right) \\
& D_{i j}=\frac{1}{3} \sum_{k=1}^{n}\left(\bar{Q}_{i j}\right)_{k}\left(z_{k}^{3}-z_{k-1}^{3}\right)
\end{aligned}
$$

where $z_{k}$ is the distance from the mid-plane to the centroid of the $k$ layer (Fig. 1) [12].

\section{Element-free Galerkin method}

Before describing the EFG method, it is present briefly the governing equations for an incompressible, elastic and 
isotropic medium. Considering the two-dimensional problem of solid mechanics $\Omega$ bounded by $\Gamma: \nabla \sigma+b=0$ in $\Omega$, where $\sigma$ is the stress tensor, which corresponds to the displacement field $\mathbf{u}=\{u v\}^{T}, \mathbf{b}$ the body force vector, and $\nabla$ is the divergence operator. The boundary conditions are given by: $\sigma \mathbf{n}=\bar{t}$ on the natural boundary $\Gamma_{t}$, and $\mathbf{u}=\overline{\mathbf{u}}$ on the essential boundary $\Gamma_{u}$, where the superposed bar denotes the prescribed traction and displacement boundary values and $\mathbf{n}$ is the unit outward vector normal to the boundary $\Gamma_{t}$.

The variational or weak form of the equilibrium equation $(\nabla \sigma+b=0)$ is posed as follows. Consider trial functions $\mathbf{u}=(\mathbf{x}) \in H^{1}$ and Lagrange multipliers $\lambda \in H^{0}$ for all test functions $\delta \mathbf{v}=(\mathbf{x}) \in H^{1}$ and $\delta \lambda \in H^{0}$, as follows [13]:

$$
\begin{aligned}
\int_{\Omega} \delta\left(\nabla_{S} \mathbf{v}^{T}\right) & \sigma d \Omega-\int_{\Omega} \delta \mathbf{v}^{T} \mathbf{b} d \Omega-\int_{\Gamma_{t}} \delta \mathbf{v}^{T} \overline{\mathbf{t}} d \Gamma \\
& -\int_{\Gamma_{u}} \delta^{T} \cdot(\mathbf{u}-\overline{\mathbf{u}}) d \Gamma-\int_{\Gamma_{u}} \delta \mathbf{v}^{T} \lambda d \Gamma=0 .
\end{aligned}
$$

Then the equilibrium (Eq. (1)) and the boundary conditions (Eqs. (2) and (3)) are satisfied. Here $\nabla_{S} \mathbf{v}^{T}$ is the symmetric part of $\nabla \mathbf{v}^{T} ; H^{1}$ and $H^{0}$ denote the Hilbert spaces of degree one and zero, respectively. It is observed that the trial functions do not satisfy the essential boundary conditions, so that they are imposed with Lagrange multipliers [2].

The EFG method employs recently developed approximation theories that permit the resultant shape functions to be constructed entirely in terms of arbitrarily placed nodes [13].

\subsection{Moving least-square approximations}

In this section a brief for moving least-square (MLS) [14] approximation is given. More details are referred to in references [2] and [13]. The moving least-squares interpolation is defined in a domain by:

$$
\mathbf{u}^{h}(\mathbf{x})=\sum_{j=1}^{n} p_{j}(\mathbf{x}) a_{j}(\mathbf{x})=\mathbf{p}^{T}(\mathbf{x}) \mathbf{a}(\mathbf{x})
$$

where $\mathbf{p}^{T}(\mathbf{x})=\left\{p_{1}(\mathbf{x}), p_{2}(\mathbf{x}), \ldots, p_{m}(\mathbf{x})\right\}$ is a vector for complete basis functions of order $m$ and $\mathbf{a}^{T}(\mathbf{x})=$ $\left\{a_{1}(\mathbf{x}), a_{2}(\mathbf{x}), \ldots, a_{m}(\mathbf{x})\right\}$ is a vector of unknown parameters that depend on $\mathbf{x}$. For example, in two dimensions with $x_{1}$ and $x_{2}$ coordinates for $m=3$ and $m=6$, representing linear and quadratic basis functions, respectively:

$$
\mathbf{p}^{T}(\mathbf{x})=\left[1 x_{1} x_{2}\right] \text { and } \mathbf{p}^{T}(\mathbf{x})=\left[1 x_{1} x_{2} x_{1}^{2} x_{1} x_{2} x_{2}^{2}\right]
$$

In equation (11), the coefficient vector $\mathbf{a}(\mathbf{x})$ is obtained at point $\mathbf{x}$ by minimizing a weighted discrete least-squares norm as follows:

$$
J=\sum_{I=1}^{n} w\left(\mathbf{x}-\mathbf{x}_{I}\right)\left[\mathbf{p}^{T}\left(\mathbf{x}_{I}\right) \mathbf{a}(\mathbf{x})-u_{I}\right]^{2}
$$

where $n$ is the number of points in the neighbourhood of $\mathbf{x}$ (called the domain of influence of $\mathbf{x}$ ) for which the weight function $w\left(\mathbf{x}-\mathbf{x}_{I}\right) \neq 0$, and $u_{I}$ is the nodal value of $\mathbf{u}$ at $\mathbf{x}=\mathbf{x}_{I}$.

The stationarity of $J$ with respect to $\mathbf{a}(\mathbf{x})$ leads to the following linear relation:

$$
\mathbf{A}(\mathbf{x}) \mathbf{a}(\mathbf{x})=\mathbf{B}(\mathbf{x}) \mathbf{u}, \text { or } \mathbf{a}(\mathbf{x})=\mathbf{A}^{-1}(\mathbf{x}) \mathbf{B}(\mathbf{x}) \mathbf{u},
$$

where $\mathbf{A}(\mathbf{x})$ and $\mathbf{B}(\mathbf{x})$ are the matrices defined by:

$$
\mathbf{A}(\mathbf{x})=\sum_{I=1}^{n} w\left(\mathbf{x}-\mathbf{x}_{I}\right) \mathbf{p}\left(\mathbf{x}_{I}\right) \mathbf{p}^{T}\left(\mathbf{x}_{I}\right)
$$

$$
\begin{aligned}
\mathbf{B}(\mathbf{x})=\left[w\left(\mathbf{x}-\mathbf{x}_{1}\right) \mathbf{p}\left(\mathbf{x}_{1}\right) w\left(\mathbf{x}-\mathbf{x}_{2}\right)\right. & \\
& \left.\times \mathbf{p}\left(\mathbf{x}_{2}\right) w \ldots\left(\mathbf{x}-\mathbf{x}_{n}\right) \mathbf{p}\left(\mathbf{x}_{n}\right)\right], \\
\mathbf{u}^{T}= & {\left[\begin{array}{lllll}
u_{0} & u_{1} & u_{2} & \ldots & u_{n}
\end{array}\right] . }
\end{aligned}
$$

Substituting (14) in (11), it is possible to write:

$$
\mathbf{u}^{h}(\mathbf{x})=\sum_{I=1}^{n} \Phi_{I}(\mathbf{x}) u_{I}=\Phi(\mathbf{x}) \mathbf{u}
$$

where the MLS shape function $\Phi_{I}(\mathbf{x})$ is defined by:

$$
\Phi_{I}(\mathbf{x})=\sum_{j=0}^{m} p_{j}(\mathbf{x})\left(\mathbf{A}^{-1}(\mathbf{x}) \mathbf{B}(\mathbf{x})\right)_{j I}=\mathbf{p}^{T} \mathbf{A}^{-1} \mathbf{B}_{I}
$$

The partial derivatives of $\Phi_{I}(\mathbf{x})$ can be obtained as follows:

$\Phi_{I, i}(\mathbf{x})=\sum_{j=0}^{m}\left\{p_{j, i}\left(\mathbf{A}^{-1} \mathbf{B}\right)_{j I}+p_{j}\left(\mathbf{A}_{, i}^{-1} \mathbf{B}+\mathbf{A}^{-1} \mathbf{B}_{, i}\right)_{j I}\right\}$,

where: $\mathbf{A}_{, i}^{-1}=-\mathbf{A}^{-1} \mathbf{A}_{, i} \mathbf{A}^{-1}$ in which ()$_{, i}=\partial() / \partial x_{i}$.

\subsection{Shape functions}

The EFG method modifies the weight functions to construct an approximation which is capable of reproducing the linear basis (Eq. (2)). This approximation is based upon MLS in curve and surface fitting [14].

The shape function in equation (19) is constructed as:

$$
\Phi_{I}(\mathbf{x})=\gamma^{T}(\mathbf{x}) \mathbf{B}_{I}(\mathbf{x})
$$

in which:

$$
\gamma(\mathbf{x})=\mathbf{A}(\mathbf{x})^{-1} \mathbf{p}(\mathbf{x}) .
$$

The derivatives of $\gamma(\mathbf{x})$ are computed similarly:

$$
\gamma_{, x}(\mathbf{x})=\mathbf{A}^{-1}\left(\mathbf{p}_{, x}-\mathbf{A}_{, x} \gamma\right) .
$$

The vector $\gamma(\mathbf{x})$ is determined using an LU decomposition of $\mathbf{A}$ followed by back substitution, leading to a computationally efficient procedure for computing the derivates of $\mathbf{u}^{h}(\mathbf{x})$. 
In this work, it is used the following cubic spline weighting function [13]:

$$
w(r)= \begin{cases}\frac{2}{3}-4 r^{2}+4 r^{3} & \text { for } r \leqslant \frac{1}{2} \\ \frac{4}{3}-4 r+4 r^{2}-\frac{4}{3} r^{3} & \text { for } \frac{1}{2}<r \leqslant 1 \\ 0 & \text { for } r>1\end{cases}
$$

in which $r$ is the radius of the domain of influence. The tensor product of weighting functions at any point is defined as:

$$
w\left(\mathbf{x}-\mathbf{x}_{I}\right)=w\left(r_{x}\right) w\left(r_{y}\right)
$$

where $w\left(r_{x}\right)$ or $w\left(r_{y}\right)$ is given by equation (24) with $r$ replaced by $r_{x}$ or $r_{y}$ respectively. These parameters are:

$$
r_{x}=\frac{\left|\mathbf{x}-\mathbf{x}_{I}\right|}{d_{m x}}, \quad r_{y}=\frac{\left|\mathbf{y}-\mathbf{y}_{I}\right|}{d_{m y}}
$$

where $d_{m x}=d_{\max } c_{x I}$ and $d_{m y}=d_{\max } c_{y I}$. The parameter $d_{\text {max }}$ is a scaling parameter which is typically $2.0-4.0$ for static analysis and $c_{x I}$ and $c_{y I}$ are determined at a particular node by searching for enough neighbor nodes such that $\mathbf{A}$ is non-singular everywhere in the domain, and thus invertible [15]. If the nodes are uniformly spaced, the values $c_{x I}$ and $c_{y I}$ correspond to the distance between the nodes in the $x$ and $y$ directions, respectively.

\subsection{Discrete equations}

In order to obtain the discrete equations form the weak form (Eq. (10)) the approximate solution $\mathbf{u}$ and test function $\delta \mathbf{v}$ are constructed according to equation (16). The Lagrange multiplier $\lambda$ is expressed by [15]:

$$
\begin{gathered}
\lambda=\sum_{K} \psi_{K}(s) \lambda_{K}, \\
\delta \lambda=\sum_{K} \psi_{K}(s) \delta \lambda_{K},
\end{gathered}
$$

where $\psi_{k}(s)$ is a Lagrange interpolant and $s$ is the arclength along the boundary; the repeated indices designate summations. The final discrete equations can be obtained by substituting the trial functions, test functions and equations (27) and (28) into the weak form (Eq. (10)), yielding the following system of linear algebraic equations [15] for static analysis:

$$
\left[\begin{array}{ll}
\mathbf{K} & \mathbf{G} \\
\mathbf{G}^{T} & 0
\end{array}\right]\left\{\begin{array}{l}
\mathbf{q} \\
\lambda
\end{array}\right\}=\left\{\begin{array}{l}
\mathbf{g} \\
\mathbf{h}
\end{array}\right\}
$$

and for modal analysis [11]:

$$
\left(\left[\begin{array}{ll}
\mathbf{K} & \mathbf{G} \\
\mathbf{G}^{T} & 0
\end{array}\right]-\left\{\begin{array}{l}
\mathbf{f} \\
\lambda
\end{array}\right\}\left[\begin{array}{ll}
\mathbf{M} & \mathbf{G} \\
\mathbf{G}^{T} & 0
\end{array}\right]\right)\left\{\begin{array}{l}
\boldsymbol{\Delta} \\
\lambda
\end{array}\right\}=\{\mathbf{0}\}
$$

where $\mathbf{K}, \mathbf{M}$ and $\mathbf{f}$ are the stiffness matrix, mass matrix and applied forces vector, respectively:

$$
\begin{aligned}
\mathbf{K}_{I J} & =\int_{\Omega} \mathbf{B}_{I}^{T} \mathbf{D B}_{J} d \Omega \\
\mathbf{M}_{I J} & =\int_{\Omega} \rho \Phi_{I} \Phi_{J} d \Omega \\
\mathbf{G}_{I K} & =-\int_{\Gamma_{u t}} \Phi_{i} \psi_{K} \mathbf{S} d \Gamma \\
\mathbf{g}_{I} & =\int_{\Gamma_{t}} \Phi_{I} \overline{\mathbf{t}} d \Gamma+\int_{\Omega} \Phi_{I} \mathbf{b} d \Omega \\
\mathbf{h}_{K} & =-\int_{\Gamma_{u t}} \psi_{K} \mathbf{S} \overline{\mathbf{u}} d \Gamma
\end{aligned}
$$

where $\mathbf{S}$ is a diagonal matrix $\left(\mathbf{S}_{I I}=1\right.$ if the displacement is imposed in $\mathbf{x}_{I}$ and 0 in other case [16]) and $\rho$ is the density of material.

The differential operator matrix for two dimensions case is:

$$
\mathbf{B}_{I}=\left[\begin{array}{ll}
\Phi_{I, x} & 0 \\
0 & \Phi_{I, y} \\
\Phi_{I, y} & \Phi_{I, x}
\end{array}\right]
$$

For isotropic material [10]:

$$
\mathbf{D}=\frac{E}{1-\nu^{2}}\left[\begin{array}{ccc}
1 & \nu & 0 \\
\nu & 1 & 0 \\
0 & 0 & \frac{1-\nu}{2}
\end{array}\right], \text { for plane stress }
$$

$\mathrm{D}=$

$$
\frac{E}{(1+\nu)(1-2 \nu)}\left[\begin{array}{ccc}
1-\nu & \nu & 0 \\
\nu & 1-\nu & 0 \\
0 & 0 & \frac{1-2 \nu}{2}
\end{array}\right] \text {, for plane strain. }
$$

For orthotropic material D is given by equation (9) [11].

In order to perform the numerical integration a background mesh is needed, which can be independent of the arrangement of meshless nodes. Standard Gaussian quadratures were used to evaluate the integrals for assembling the stiffness, mass matrix and force vector.

\section{Reliability index estimation as a general optimization problem}

In traditional deterministic design optimization, the optimization problem is generally formulated in the physical space of the design variables and consists in minimizing or maximizing an objective function subject to geometrical, physical or functional constraints in the form:

$$
\min f(\{y\})
$$


subjected to $g_{k}(\{y\}) \leqslant 0$, where $\{y\}$ designates the vector of deterministic design variables.

In reliability analysis, which involves random variables $\{x\}$, the deterministic optimal solution is not considered the exact solution of the optimum design but is one of the most probably design. In this case, the failure surface or limit state function is given by $G(\{x\},\{y\})=0$. This surface defines the limit between the safe region $G(\{x\},\{y\})>0$ and unsafe region of the design space. The failure occurs when $G(\{x\},\{y\})<0$, and the failure probability is calculated as $P_{f}=\operatorname{prob}[G(\{x\},\{y\}) \leqslant 0]$.

The reliability index $\beta$ is introduced as a measure of the reliability level of the system and is estimated in the so-called reduced coordinate system, where the random variables $\{u\}$ are statistically independent with zero mean and unit standard deviation. Thus a pseudo-probabilistic transformation $\{u\}=T[\{x\},\{y\}]$ must be defined for mapping the original space into the reduced coordinate system (see [17]). Considering that the probability density in the reduced space decays exponentially with the distance from the origin of this space, the point with maximum probability of failure (most probable point) on the limit state surface is the point of minimum distance from the origin. The reliability index is thus defined as the minimum distance between the origin of the reduced space and the hyper surface representing the limit state function $H(\{u\},\{y\})$. Hence, it is possible to find the most probable point or design point by solving a constrained optimization problem that is [18]:

$$
\beta=\min \sqrt{\sum_{i=1}^{n} u_{i}^{2}}
$$

subjected to safety constraints:

$$
H(\{u\},\{y\})=0 .
$$

By formally introducing a cumulative density function $(\phi)$ of the normal probability distribution function, the first order approximation (tangent plane at the most probable point-MPP) to $P_{f}$ can be written as [18]:

$$
P_{f}=1-\phi(\beta) .
$$

In order to estimate the reliability index (Eq. (40)) it is possible make use of a variety of optimization methods.

FORM and SORM can be considered as gradientbased methods since they demand the evaluation of the partial derivatives of the limit state function with respect to the random variables at each iteration step.

FORM is based on linear (first order) approximation of the limit state surface tangent to the most probable point of the failure surface to the origin of a reduced coordinate system. Thus, the random variables are transformed to reduced variables in a reduced coordinate system. For estimating the reliability index based on FORM one can use the algorithm suggested in [19] in which the limit state function does not need to be solved because a N-R type recursive algorithm is introduced to find the design point [20]. This algorithm has been widely used in the literature [18] and used in this study.

SORM estimates the probability of failure by using a nonlinear approximation of the limit state function by a second order representation. The curvatures of the limit state function are approximated by the secondorder derivatives with respect to the original variables. Thus, SORM improves FORM by including additional information about the curvature of the limit state function through a curvature parameter. SORM was explored in [21] using quadratic approximations. In that work the authors use a simple closed-form solution for the computation of failure probability using a second-order approach given by [22] based on the theory of asymptotic approximation. The SORM suggested by [22] is employ in this work. SORM uses as initial value the reliability index value estimated through FORM. Zhao and Ono [23] give more details of these classical techniques.

However, the solution of the optimization problem given by equation (40) by using classical gradient-based optimization methods, as a N-R, is not a simple task due to the existence of local minima in the design space and the necessity of computation of the gradients (partial derivatives). The existence of multiple MPPs is similar to multiple local minima in optimization. The solutions of many problems in structural optimization can be considered to be satisfactory once a local minimum is reached. However, this is an unacceptable procedure in reliability analysis since the local MPP may not represent the worst failure scenario and the actual failure may occur below the predicted level. Hence, only the global MPP represents the actual structural reliability [24].

Another difficulty that must be remembered is that traditional methods FORM and SORM require an initial guess of the solution (reliability index and random variables) and it is not always possible to assure global convergence. These aspects motivated the authors of this paper to explore an alternative approach for estimation of reliability index, which do not require the computation of gradients of the limit state function and are intrinsically based on multidirectional search. This approach was proposed initially in [3]. In this work the authors use the approach that uses finite element analysis and EFG method to evaluate implicit limit state functions and is based on a heuristic based reliability method (HBRM), which allows the use of optimization methods such as genetic algorithms $[4,5]$, particle swarm optimization $[6,7]$ and ant colony optimization $[8,9]$. It is believed that such approach can circumvent some of the difficulties mentioned above, and thus lead to improved results of reliability analysis.

Taking into account the performance of HBRM, it was observed that this methodology is able to handle multiple limit state functions based on numerical models and probabilistic variables related to geometrical, load and material properties parameters. Rojas et al. $[3,25,26]$ give more details of HBRM. In this work was used GA algorithm in HBRM. The following section discusses the main ideas about this heuristic technique. 

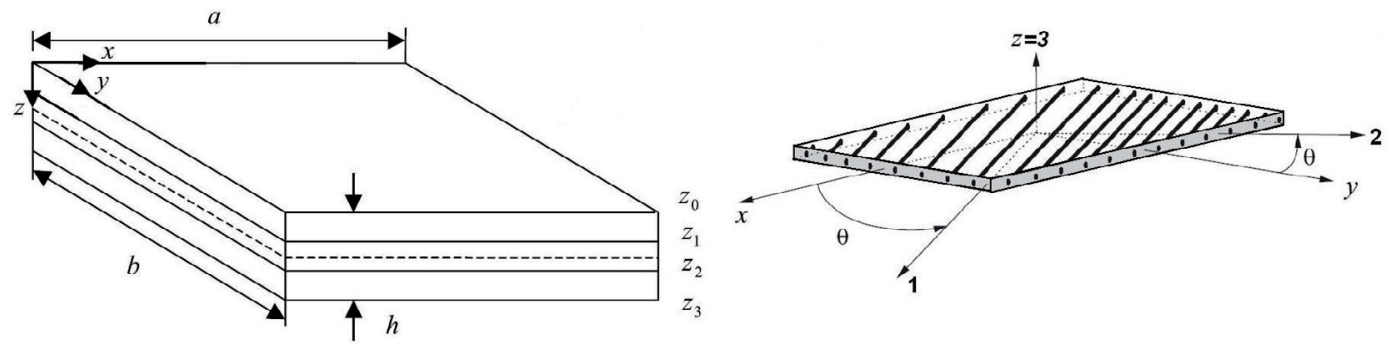

Fig. 2. Laminated composite plate.

\section{Genetic algorithms}

\subsection{Back to nature}

GA is an optimization algorithm used to find approximate solutions to difficult-to-solve problems through application of the principles of evolutionary biology to computer science. GA uses biologically-derived techniques such as inheritance, mutation, natural selection, and recombination (or crossover).

GA is based on Darwin's theory of survival and evolution of species, as explained in [4] and [5]. The algorithm starts from a population of random individuals, viewed as candidate solutions to the problem. During the evolutionary process, each individual of the population is evaluated, reflecting its adaptation capability to the environment. Some of the individuals of the population are preserved while others are discarded; this process mimics the natural selection in the Darwinism. The remaining group of individuals is paired in order to generate new individuals to replace the worst ones in the population, which are discarded in the selection process. Finally, some of them can be submitted to mutation, and as a consequence, the chromosomes of these individuals are altered. The entire process is repeated until a satisfactory solution is found.

\subsection{Algorith description}

The outline of a basic GA is as follows:

1. define the GA parameters (population size, selection method, crossover method, mutation rate, etc.);

2. create an initial population (it just allocates memory);

3. evaluate the objective function and take it as a fitness measure of each individual;

4. select the mates to the crossover;

5. reproduce and replace the worst individuals of the population;

6. mutate to avoid premature convergence;

7. go to step 3 and repeat until the stop criteria is achieved.

Although the initially proposed GA algorithm was dedicated to discrete variables only, nowadays improvements are available to deal with discrete and continuous variables. To obtain more information about GA see $[4,5]$.
Table 1. Geometrical properties of laminated composite plate.

\begin{tabular}{|c|c|c|c|c|c|}
\hline $\begin{array}{l}L(\mathrm{~m}) \\
\end{array}$ & $H(\mathrm{~m})$ & $h(\mathrm{~m})$ & $t(\mathrm{~m})$ & $\theta$ & $N_{x}(\mathrm{~N} / \mathrm{m})$ \\
\hline 5 & 5 & 0.4 & 0.1 & $-303030-30$ & $1 \times 10^{6}$ \\
\hline
\end{tabular}

Table 2. Mechanical properties of laminated composite plate.

\begin{tabular}{cccc}
\hline$E_{1}(\mathrm{~Pa})$ & $E_{2}(\mathrm{~Pa})$ & $G_{12}(\mathrm{~Pa})$ & $\nu_{12}$ \\
\hline $4.5 \times 10^{10}$ & $1.2 \times 10^{10}$ & $4.5 \times 10^{9}$ & 0.3 \\
\hline
\end{tabular}

Table 3. Displacements for EFG and FEM methods of the laminated composite plate.

\begin{tabular}{cccc}
\hline & EFG & FEM & Error (\%) \\
\hline$u_{x}(\mathrm{~m})$ & $5.05 \times 10^{-4}$ & $5.05 \times 10^{-4}$ & 0.006 \\
$u_{y}(\mathrm{~m})$ & $-3.43 \times 10^{-4}$ & $-3.43 \times 10^{-4}$ & $6.41 \times 10^{-6}$ \\
\hline
\end{tabular}

Table 4. Statistical parameters of design variables of the laminated composite plate, case 1 .

\begin{tabular}{llcc}
\hline & Distribution & Mean & CV \\
\hline$h(\mathrm{~m})$ & Normal & 0.4 & $10 \%$ \\
$\theta\left({ }^{\circ}\right)$ & Normal & 30 & $10 \%$ \\
$E_{1}\left(\mathrm{~N} / \mathrm{m}^{\wedge} 2\right) \times 10^{10}$ & Lognormal & 4.5 & $10 \%$ \\
$E_{2}\left(\mathrm{~N} / \mathrm{m}^{\wedge} 2\right) \times 10^{10}$ & Lognormal & 1.2 & $10 \%$ \\
$\nu_{12}$ & Lognormal & 0.30 & $10 \%$ \\
$G_{12}\left(\mathrm{~N} / \mathrm{m}^{\wedge} 2\right) \times 10^{9}$ & Lognormal & 4.5 & $10 \%$ \\
$N_{x}(\mathrm{~N} / \mathrm{m}) \times 10^{6}$ & Lognormal & 1 & $10 \%$ \\
\hline
\end{tabular}

\section{Numerical applications}

Consider a laminated composite rectangular plate illustrated in Figure 2 and which geometric and mechanical properties are summarized in Tables 1 and 2, respectively.

The boundary conditions include: essential boundary conditions on the bottom $\left(u_{y}=0\right)$ and left edges $\left(u_{x}=0\right)$ and natural boundary conditions on the right edge on which traction $N_{x}$ is applied.

In this case: $\nu_{21}=\nu_{12} E_{2} / E_{1}$.

For integration procedure a $4 \times 4$ Gauss quadrature is employed for a circular domain of influence. Concerning weight functions, the cubic spline function is used. The essential boundary condition is imposed using Lagrange interpolants.

Figure 3 illustrates Gauss points and deformed configuration of laminated composite plate. 

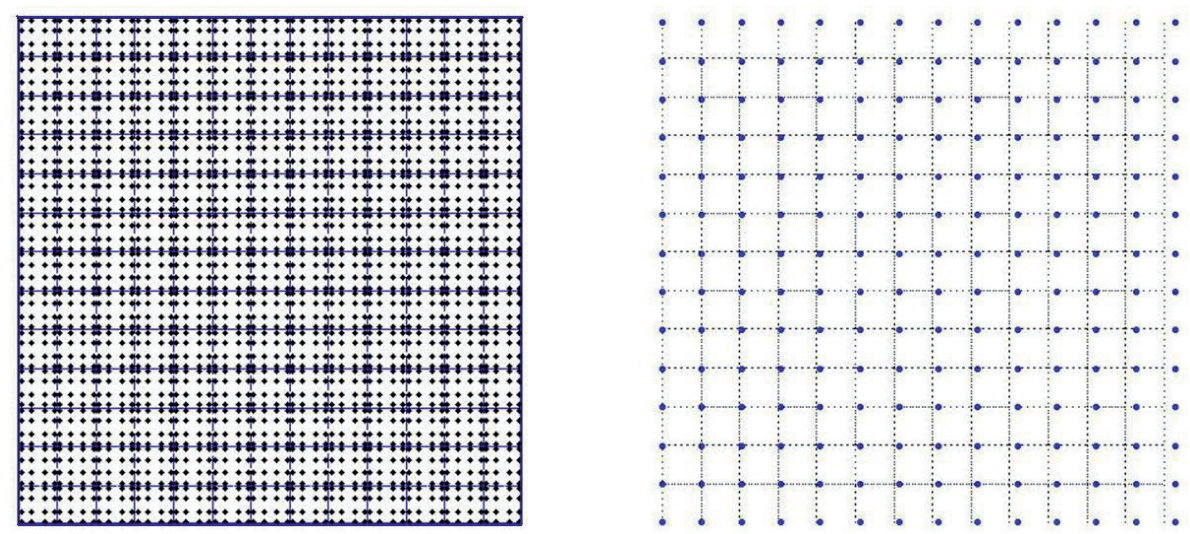

Fig. 3. Gauss points and deformed configuration of laminated composite plate.
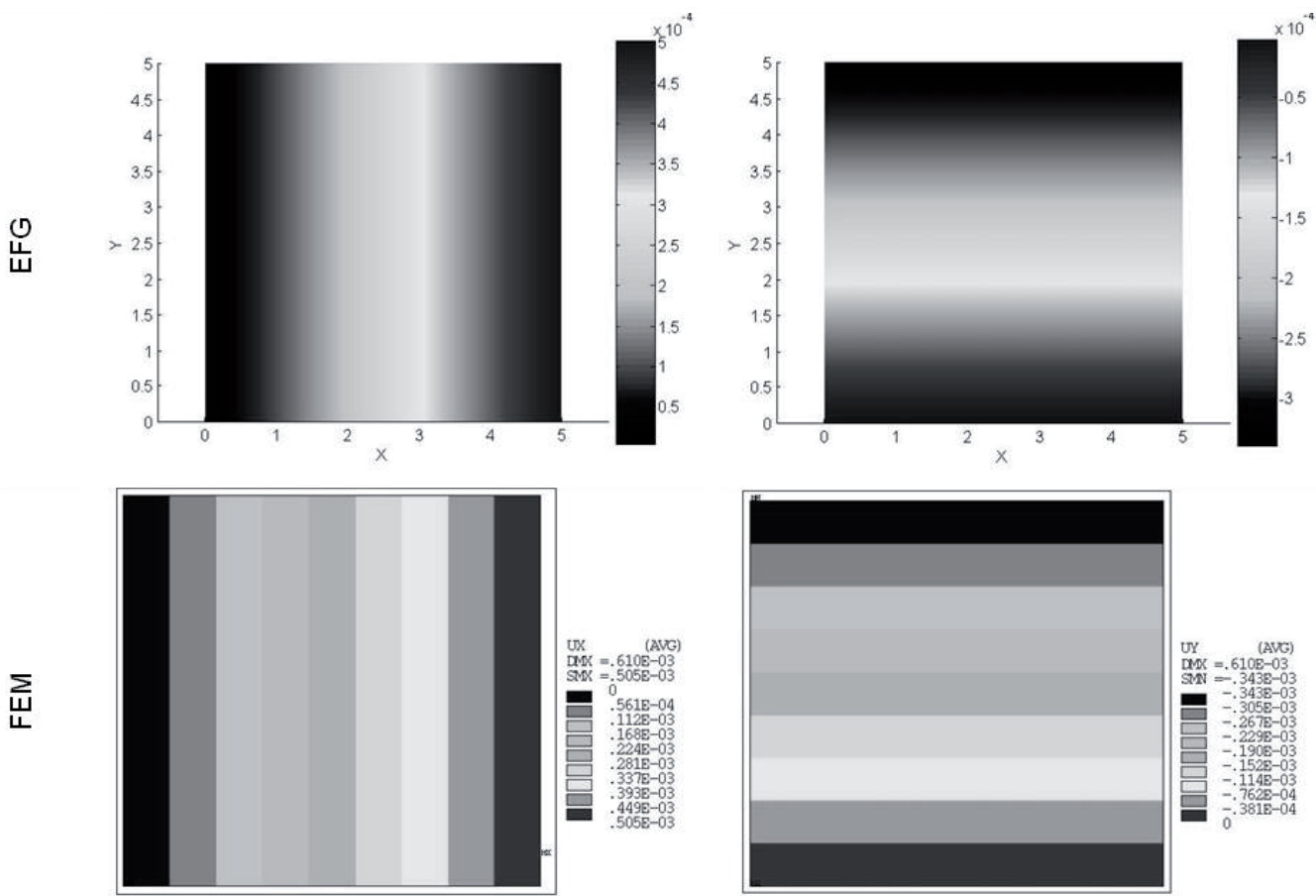

$u_{x}$

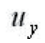

Fig. 4. M-H loop of the film.

The horizontal and vertical displacements were obtained using EFG and FEM analysis (Fig. 4). Numerical simulations through MEF were estimated using PLANE42 finite element of ANSYS ${ }^{\circledR}$.

A comparison between maximal displacements obtained by EFG and FEM approaches are exposed in Table 6 as well as its differences. Taking to account these results it is possible to validate the EFG model.

Two cases are proposed for reliability analysis, the case 1 consider 7 design variables and case 2 consider 12 design variables in laminated composite plate. The statistical parameters (mean and coefficient of variation) of design variables for the first case are summarized in Table 4 .
The limit state function is defined by the probability of failure $P_{f}\left[u_{x}\left(x_{i}\right) \leqslant u_{x}^{\lim }\right]$, where $x_{i}$ are the design variables and $u_{x}^{\lim }=1 \times 10^{-3} \mathrm{~m}$.

Table 5 summarizes the results obtained by N-R in FORM and SORM approaches and GA in HBRM. The limit state function is evaluated by FEM and EFG methods. The number of evaluations of the limit state functions for FORM and SORM (until convergence) and for HBRM (until stop criterion) is represented by $N_{G}$.

The results calculated by FORM and SORM are better than HBRM counterparts for the same number of evaluations of limit state function using EFG and FEM approaches. For all reliability approaches, EFG and FEM analysis present close results. HBRM results can be taking as an initial guess to FORM and SORM procedures [27]. 
Table 5. Reliability analysis results, case 1.

\begin{tabular}{lcccccc}
\hline & \multicolumn{3}{c}{ EFG } & & & FEM \\
\hline & FORM & SORM & HBRM & FORM & SORM & HBRM \\
\hline$h(\mathrm{~m})$ & 0.32 & 0.32 & 0.39 & 0.32 & 0.32 & 0.42 \\
$\theta\left({ }^{\circ}\right)$ & 35.85 & 35.85 & 29.03 & 35.91 & 35.91 & 29.50 \\
$E_{1}\left(\mathrm{~N} / \mathrm{m}^{2}\right) \times 10^{10}$ & 4.20 & 4.20 & 4.86 & 4.21 & 4.21 & 4.60 \\
$E_{2}\left(\mathrm{~N} / \mathrm{m}^{2}\right) \times 10^{10}$ & 1.19 & 1.19 & 1.30 & 1.19 & 1.19 & 1.07 \\
$\nu_{12}$ & 0.30 & 0.30 & 0.32 & 0.30 & 0.30 & 0.28 \\
$G_{12}\left(\mathrm{~N} / \mathrm{m}^{2}\right) \times 10^{9}$ & 4.09 & 4.09 & 4.53 & 4.08 & 4.08 & 4.36 \\
$N_{x}(\mathrm{~N} / \mathrm{m}) \times 10^{6}$ & 1.16 & 1.16 & 0.99 & 1.16 & 1.16 & 1.10 \\
$N_{G}$ & 49 & 86 & 100 & 49 & 86 & 100 \\
$\beta$ & 3.38 & 3.33 & 1.38 & 3.38 & 3.38 & 1.74 \\
$\operatorname{Pf}(\%)$ & 0.04 & 0.04 & 8.45 & 0.04 & 0.04 & 4.07 \\
$\operatorname{Reliability~}(\%)$ & 99.96 & 99.96 & 91.55 & 99.96 & 99.96 & 95.93 \\
\hline
\end{tabular}

Table 6. Statistical parameters of design variables of the laminated composite plate, case 2.

\begin{tabular}{lccc}
\hline & Distribution & Mean & CV \\
\hline$t_{1}(\mathrm{~m})$ & Normal & 0.1 & $10 \%$ \\
$t_{2}(\mathrm{~m})$ & Normal & 0.1 & $10 \%$ \\
$t_{3}(\mathrm{~m})$ & Normal & 0.1 & $10 \%$ \\
$t_{4}(\mathrm{~m})$ & Normal & 0.1 & $10 \%$ \\
$\theta_{1}\left({ }^{\circ}\right)$ & Normal & -30 & $10 \%$ \\
$\theta_{2}\left({ }^{\circ}\right)$ & Normal & 30 & $10 \%$ \\
$\theta_{3}\left({ }^{\circ}\right)$ & Normal & 30 & $10 \%$ \\
$\theta_{4}\left({ }^{\circ}\right)$ & Normal & -30 & $10 \%$ \\
$E_{1}\left(\mathrm{~N} / \mathrm{m}^{2}\right)$ & Lognormal & $4.5 \times 10^{10}$ & $10 \%$ \\
$E_{2}\left(\mathrm{~N} / \mathrm{m}^{2}\right)$ & Lognormal & $1.2 \times 10^{10}$ & $10 \%$ \\
$G_{12}\left(\mathrm{~N} / \mathrm{m}^{2}\right)$ & Lognormal & $4.5 \times 10^{9}$ & $10 \%$ \\
$N_{x}\left(\mathrm{~N} / \mathrm{m}^{2}\right)$ & Lognormal & $1 \times 10^{6}$ & $10 \%$ \\
\hline
\end{tabular}

Table 7. Reliability analysis results, case 2 .

\begin{tabular}{lcccccc}
\hline & \multicolumn{3}{c}{ EFG } & & & FEM \\
\hline & FORM & SORM & HBRM & FORM & SORM & HBRM \\
\hline$t_{1}(\mathrm{~m})$ & 0.09 & 0.09 & 0.09 & 0.09 & 0.09 & 0.10 \\
$t_{2}(\mathrm{~m})$ & 0.09 & 0.09 & 0.11 & 0.09 & 0.09 & 0.10 \\
$t_{3}(\mathrm{~m})$ & 0.09 & 0.09 & 0.10 & 0.09 & 0.09 & 0.10 \\
$t_{4}(\mathrm{~m})$ & 0.09 & 0.09 & 0.10 & 0.09 & 0.09 & 0.10 \\
$\theta_{1}\left(^{\circ}\right)$ & -32.64 & -32.64 & -31.46 & -33.42 & -33.42 & -33.74 \\
$\theta_{2}\left({ }^{\circ}\right)$ & 33.24 & 33.24 & 30.92 & 32.62 & 32.62 & 30.89 \\
$\theta_{3}\left({ }^{\circ}\right)$ & 33.24 & 33.24 & 29.94 & 32.62 & 32.62 & 32.76 \\
$\theta_{4}\left({ }^{\circ}\right)$ & -32.64 & -32.64 & -29.29 & -33.42 & -33.42 & -32.21 \\
$E_{1}\left(\mathrm{~N} / \mathrm{m}^{2}\right) \times 10^{10}$ & 3.80 & 3.80 & 4.47 & 3.82 & 3.82 & 4.18 \\
$E_{2}\left(\mathrm{~N} / \mathrm{m}^{2}\right) \times 10^{10}$ & 1.19 & 1.19 & 1.28 & 1.20 & 1.20 & 1.31 \\
$G_{12}\left(\mathrm{~N} / \mathrm{m}^{2}\right) \times 10^{9}$ & 3.84 & 3.84 & 5.03 & 3.82 & 3.82 & 4.28 \\
$N_{x}(\mathrm{~N} / \mathrm{m}) \times 10^{6}$ & 1.37 & 1.37 & 0.98 & 1.36 & 1.36 & 0.99 \\
$N_{G}$ & 73 & 165 & 100 & 73 & 165 & 100 \\
$\beta$ & 4.74 & 4.72 & 1.99 & 4.72 & 4.72 & 2.23 \\
Pf $(\%)$ & $1.08 \times 10^{-4}$ & $1.18 \times 10^{-4}$ & 2.32 & $1.16 \times 10^{-4}$ & $1.16 \times 10^{-4}$ & 1.30 \\
Reliability $(\%)$ & 100 & 100 & 97.68 & 100 & 100 & 98.70 \\
\hline
\end{tabular}

Table 6 summarizes the statistical parameters of the design variables considered in the second case (12 design variables). In this table, $t_{i}$ and $\theta_{i}$ represent the thickness and fibre orientation of each layer of the laminated composite plate.

Reliability results obtained by FORM, SORM and HBRM using FEM and EFG methods for case 2 are summarized in Table 7 .
Similar to case 1, FORM and SORM results are considered better than HBRM counterparts for the same number of evaluations of limit state function. In this case, the results obtained by EFG are better than FEM counterparts using FORM, very close using SORM but not using HBRM, where FEM have better performance in comparison with EFG. HBRM results can be used as initial guess to FORM and SORM algorithms. It is possible 
to obtain better results through HBRM for more evaluations of limit sate function.

\section{Conclusions}

A structural reliability methodology that couples FORM, SORM and HBRM with element-free Galerkin method to analyse laminated composite plates was suggested. Numerical examples on linear elasticity were used to illustrate the potentiality of the proposed procedure. Load, material and geometrical proprieties were considered as design parameters in numerical applications. Good agreement was observed between the results of the EFG method allied to RA. HBRM algorithm is not competing with traditional methods FORM and SORM, but it was tested because is able to solve global optimization problems efficiently. The obtained results demonstrate that the predicted reliability levels are accurate in comparison with similar approach that uses FEM to evaluate implicit limit state functions. Considering the results obtained it is possible to conclude that the methodology presented can be used in more complex applications, since mesh generation of these structures can be far more time-consuming and costly than the solution of a discrete set of equations. The EFG method provides an attractive alternative to FEM analyses in evaluating limit state functions in stochastic mechanics problems and encourage authors for applied this approach in problems where finite element analysis presents some difficulties.

Msc. Rojas are thankful to CAPES Foundation (Brazilian Ministry of Education) and Programme $\mathrm{Al} \beta$ an, the European Union Programme of High Level Scholarships for Latin America, for the support to their scholarship No. E06D103742BO. Dr. Rade gratefully acknowledges $\mathrm{CNPq}$ for the support to their research activities.

\section{References}

1. S. Rahman, B.N. Rao. International Journal of Solids and Structures 38, 9313 (2001)

2. T. Belytschko, Y.Y. Lu, L. Gu. International Journal of Numerical Methods in Engineering 37, 229 (1994)

3. J.E. Rojas, F.A.C. Viana, D.A. Rade, 7 th Multidisciplinary International Conference Quality and Reliability (Tangier, Morocco, March 20-22, 2007)

4. Z. Michalewicz, Genetic Algorithms + Data Structures = Evolution Programs, 2nd edn. (Springer-Verlag, New York, USA, 1994)

5. R.L. Haupt, S.E. Haupt, Pratical Genetic Algorithms, 2nd edn. (Wiley-Interscience Publication, New York, USA, 2004)

6. J. Kennedy, R.C. Eberhart, Particle Swarm Optimization, Proceedings of the 1995 IEEE International Conference on Neural Networks (Perth, Australia, 1995), pp. 1942-1948
7. G. Venter, J.S. Sobieski, Multidisciplinary Optimization of a Transport Aircraft Wing using Particle Swarm Optimization, Proceedings of the 9th AIAA/ISSMO Symposium on Multidisciplinary Analysis and Optimization (Atlanta, GA, September 4-6, 2002), Vol. AIAA. pp. 2002-5644

8. M. Dorigo, Optimization, Learning and Natural Algorithms, Ph.D. Dissertation, Politecnico di Milano, Italy, 1992

9. K. Socha, M. Dorigo, ACO for Continuous and Mixed-Variable Optimization. Proceedings of the ANTS 2004 - Fourth International Workshop on Ant Colony Optimization and Swarm Intelligence, Brussels, Belgium, 2004

10. R.M. Jones, Mechanics of Composite Materials, 2nd edn. (Taylor and Francis, Philadelphia, 1999)

11. X.L. Chen, G.R. Liu, S.P. Lim. Composite Structures 59, $279(2003)$

12. J. Belinha, L.M.J.S. Dinis. Composite Structures 78, 337 (2007)

13. J. Dolbow, T. Belytschko. International Journal for Numerical Methods in Engineering 46, 925 (1999)

14. P. Lancaster, K. Salkauskas. Mathematics of Computation 37, 147 (1981)

15. Y.Y. Lu, T. Belytschko, L. Gu. Computer Methods in Applied Mechanics and Engineering 113, 397 (1994)

16. T. Belytschko, Y.Y. Lu, L. Gu. Engineering Fracture Mechanics 51, 295 (1995)

17. A. Mohsine, Contribution à l'optimisation Fiabiliste en Dynamique des Structures Mécaniques, Thèse de Doctorat, Institut National des Sciences Appliquées de Rouen, France, 2006

18. A. Haldar, S. Mahadevan, Reliability Assessment Using Stochastic Finite Element Analysis (John Wiley \& Sons, Inc., New York, USA, 2000)

19. R. Rackwitz, B. Fiessler. Computers and Structures 9, 484 (1978)

20. B. Fiessler, H.J. Neumann, R. Rackwitz. Journal of Engineering Mechanics. ASCE 1095, 661 (1979)

21. A. Der Kiureghian, A. De Stefano. Journal of the Engineering Mechanics Division, American Society of Civil Engineers 117 (1991)

22. K. Breitung. Journal of Engineering Mechanics ASCE 110, 357 (1984)

23. Y.-G. Zhao, T.A. Ono. Structural Safety 21, 95 (1999)

24. L. Wang, R.V. Grandhi. Comput. Struct. 59, 1139 (1996)

25. J.E. Rojas, O. Bendaou, A. Elhami, D.A. Rade, in Proceedings of the Eleventh International Conference on Civil, Structural and Environmental Engineering Computing, edited by B.H.V. Topping (Civil-Comp Press, Stirlingshire, UK, 2007), paper 43

26. J.E. Rojas, F.A.C. Viana, A. Elhami, D.A. Rade, in Proceedings of the Eleventh International Conference on Civil, Structural and Environmental Engineering Computing, edited by B.H.V. Topping (Civil-Comp Press, Stirlingshire, United Kingdom, 2007), paper 41

27. J.E. Rojas, F.A.C. Viana, D.A. Rade DA, V.Jr. Steffen, A Procedure for Structural Reliability Analysis Based on Finite Element Modeling, IV Congresso Nacional de Engenharia Mecânica, 22 a 25 de Agosto 2006, Recife-PE, Brasil, 2006 